\title{
Habituation in Stentor: Produced by Mechanoreceptor Channel Modification
}

\author{
David C. Wood \\ Department of Behavioral Neuroscience, University of Pittsburgh, Pittsburgh, Pennsylvania 15260
}

Habituation in Stentor was shown in the previous paper (Wood, 1988) to result from a progressive diminution in mechanoreceptor potential amplitude during the course of repetitive mechanical stimulation. The source of this diminution was analyzed in the present studies by eliciting mechanoreceptor currents from control and habituated cells under voltage clamp control. The $I-V$ plots obtained have a voltagedependent region between -60 and $-20 \mathrm{mV}$, and a linear voltage-independent region between -20 and $20 \mathrm{mV}$, with a reversal potential near $20 \mathrm{mV}$. The slope of the linear region did not change as a result of habituation indicating that the maximum mechanoreceptor current, and hence the total number of mechanoreceptor channels, was the same in control and habituated cells. The mechanoreceptor current reversal potential was changed by only a few millivolts, from 21.6 to $17.2 \mathrm{mV}$, leaving the driving force term for the mechanoreceptor current at resting potential relatively unchanged. On the other hand, the voltage-dependent region of the I-V plot became significantly steeper after habituation ( $9.6 \mathrm{mV} / \mathrm{e}$-fold change) compared to its value before habituation (12.3 $\mathrm{mV} / \mathrm{e}$-fold change). This shift in voltage dependence is sufficient to explain the diminution in receptor potential amplitude seen during normal recordings at resting potential.

The importance of the voltage-dependent characteristic of the mechanoreceptor channels to the habituation process was corroborated by the observation that the amplitude of receptor potentials elicited from cells depolarized by current pulses habituated to only a very limited degree while receptor potentials elicited at resting potential underwent marked habituation.

In addition, the importance of the voltage-dependent characteristic of mechanoreceptor channels was confirmed by data from a ligand-binding study. $(+)$-Tubocurarine(TC*) has previously been shown to bind selectively to the form of the mechanoreceptor channels that predominates at hyperpolarized potentials and to bind only weakly to the form that predominates at depolarized potentials (Wood, 1985). TC* binding was increased significantly in habituated animals as compared to controls when both were at normal resting potential, but not when both types of cells were depolarized

\footnotetext{
Received May 27, 1987; accepted Nov. 27, 1987.

I wish to acknowledge the excellent technical assistance of Suchin Huang and the helpful commentary of Dr. German Barrioneuvo during the preparation of this manuscript. This work was supported by PHS Grant NS 09733.

Correspondence should be addressed to Dr. David C. Wood, 410 Langley Hall, Department of Behavioral Neuroscience, University of Pittsburgh, Pittsburgh, PA 15260 .

Copyright $\odot 1988$ Society for Neuroscience $0270-6474 / 88 / 072254-05 \$ 02.00 / 0$
}

by increasing the extracellular concentration of $K^{+}$. Thus, the voltage-dependent characteristic of $\mathrm{TC}^{*}$ binding was affected by the habituation process, which suggests that the voltage-dependent shift between different conformations of the channels had been changed.

Taken collectively, these data indicate that the voltagedependent characteristic of the mechanoreceptor channels is modified by the habituation process.

The ciliate protozoan Stentor coeruleus contracts in an all-ornone fashion in response to an initial mechanical stimulus. However, during the course of repeated mechanical stimulation, the animal ccascs to respond to every stimulus and becomes progressively more unresponsive until it attains an asymptotic probability of response between 0.15 and 0.4 after $30 \mathrm{~min}$ of $1 /$ min stimulation (Wood, 1970). This decrement in response probability displays the parametric characteristics of habituation. Electrophysiological recordings reveal that the amplitude of the receptor potentials elicited by the mechanical stimulus decreases progressively during the course of the repetitive stimulation (Wood, 1971, 1988). This mechanoreceptor potential decrement is the principal, if not the sole, cause of behavioral habituation. The results reported in this paper indicate that this decrement in receptor potential amplitude results from alterations in the mechanoreceptor ionic channel.

Receptor potentials are produced by inward receptor currents, $I_{\mathrm{r}}$, carried by $\mathrm{Ca}^{2+}$ ions through mechanoreceptor channels whose conductance, $g_{r}$, is transiently increased by a mechanical stimulus provided the transmcmbranc potential is at a permissive value (Wood, 1982a). Mechanoreceptor channel conductance in the protozoan Stylonychia is similarly dependent on both mechanical stimulation and transmembrane potential (de Peyer and Machemer, 1978). This dual mechanical stimulus and voltage dependency can be adequately described by employing a simple extension of the Hodgkin-Huxley (1952) model for voltage dependency. In this model, the mechanoreceptor channels are assumed to have 2 closed conformations and 1 open one. One of the closed conformations, the $\mathrm{R}$ form, predominates at depolarized potentials and can be opened by a mechanical stimulus, while the other conformation, the $U$ form, predominates at hyperpolarized potentials and cannot be opened by a mechanical stimulus. The proportion of mechanoreceptor channels in the $\mathrm{R}$ and $\mathrm{U}$ forms is voltage-dependent and governed by the Boltzmann relation. Schematically, this model is,

$$
\mathrm{U} \rightleftharpoons \stackrel{\mathrm{V}_{\mathrm{m}}}{\rightleftharpoons} \mathrm{R} \stackrel{\text { Mechanical stimulus }}{\longrightarrow} \text { Open. }
$$

(+)-Tubocurarine (TC) has been shown to block $I_{\mathrm{r}}$ in a con- 
centration and voltage-dependent fashion (Wood, 1985). This blockade is reduced at depolarized potentials and can also be temporarily reversed by depolarizing voltage steps. Analogously, radioactively labeled TC (TC*) binds to Stentor in a voltagedependent fashion being maximal at resting potential and reduced at depolarized potentials. Both the electrophysiological and binding data are adequately described by a simple binding model in which it is assumed that TC binds preferentially to the $U$ form of the mechanoreceptor channel (Wood, 1982b). Therefore, in the studies reported below, $\mathrm{TC}^{*}$ binding is used to provide a measure of the number of channels in the $U$ form.

\section{Materials and Methods}

Animals. Stentor coeruleus of the Stella strain were taken from cultures maintained in the manner described in the preceding paper (Wood, 1988).

Materials. $(+)$-Tubocurarine di- $\left({ }^{14} \mathrm{C}\right)$ methyl ether iodide $\left(\mathrm{TC}^{*}\right)$ was obtained from Amersham/Searle (sp act, $88-112 \mathrm{mCi} / \mathrm{mmol}$ ).

Electrophysiology. Intracellular recordings were obtained from animals chilled to $8.5-10^{\circ} \mathrm{C}$. Each cell was penetrated through its frontal field with 2 microelectrodes. A third microelectrode, filled with culture medium and broken off to have a resistance less than $1 \mathrm{M} \Omega$, was positioned near the penetrated animal. Transmembranc potentials werc recorded as the potential difference between one of the intracellular microelectrodes and this extracellular electrode in order to minimize potential drops produced across the series resistance present in the culture medium.

Mechanical stimuli were produced by actuating a solenoid which moved the impaled cell $25 \mu \mathrm{m}$ or less through the culture medium (Wood, 1975). Actuation of the solenoid produced an initial displacement of the cell in one direction followed $1 \mathrm{sec}$ later by a return displacement that acted as a second mechanical stimulus. The velocity of these movements was monitored by a linear velocity transducer in series with the solenoid armature.

$I-V$ plots. After microelectrode penetration animals were allowed a 15 min rest before an initial measurement of their membrane properties was madc. Only animals having resting potentials more negative than $-45 \mathrm{mV}$, action potentials exceeding $65 \mathrm{mV}$ in amplitude, input resistances greater than $10 \mathrm{M} \Omega$, and mechanoreceptor potentials exceeding $5 \mathrm{mV}$ were retained for further study. An assessment of the voltage dependence of the mechanoreceptor current was then made while the cells were voltage clamped. On individual trials, the cell membrane was stepped from the $-50 \mathrm{mV}$ holding potential to a voltage between -70 and $+20 \mathrm{mV} 800 \mathrm{msec}$ before the mechanical stimulus was applied. The elicited receptor currents were brief $(<50 \mathrm{msec})$ compared to the changes in the voltage-dependent outward current on which they were superimposed and were therefore easily quantified (see Wood, 1982a, 1985 , for sample records). Each cell was tested at 4 to 10 different transmembrane potentials that were separated by multiples of $10 \mathrm{mV}$. Tests were presented only once every $3 \mathrm{~min}$ to avoid habituating the animal with the tcst stimuli. The magnitude of the $I_{\mathrm{r}}$ was plotted against the step potential at which it was elicited to generate the I-V plots. After completion of this initial I-V sequence a 15 min rest period was employed to allow for recovery from any habituating effect of the test stimuli. Habituation was then produced with a sequence of $151 / \mathrm{min}$ mechanical stimuli each of which was followed in $200 \mathrm{msec}$ by a $1 \mathrm{sec}$ depolarizing current pulse sufficient to elicit an action potential (Wood, 1988). After the $15^{\text {th }}$ trial this $1 / \mathrm{min}$ combined mechanical and electrical stimulation was maintained, except on every third trial when the cell was voltage clamped and subjected to one of the voltage step-mechanical stimulus sequences used in generating the initial I-V plot data. Each of the voltage step-mechanical stimulus sequences used before habituation was used again after habituation. Following completion of this test sequence, a $15 \mathrm{~min}$ rest was allowed before a mechanical stimulus was applied to assess recovery from habituation. Resting potential, input resistance, and action potential amplitude were reassessed prior to termination of an experiment.

The experimental sequence outlined above required in excess of $2 \mathrm{hr}$ to perform. Of the 26 penetrations meeting the initial electrophysiological criteria, only 13 survived the initial I-V testing and habituating stimuli without marked loss of resting potential, input resistance, or action potential amplitude due to damage to the cell or loss of microelec- trode penetration. Useful data for the comparison of resting potentials, input resistances, mechanoreceptor current reversal potential, or mechanoreceptor channel voltage dependence before and after habituation were obtained from all of these cells, but all of these parameters were obtained on only 2 cells. In the electrophysiological results reported below, data from a given cell are included only if comparable measurements on a given parameter were obtained both before and after application of the habituating stimuli and if the cell's resting potential, input resistance, and action potential amplitude were relatively constant up to the time of parameter measurement. These criteria were met by differing numbers of cells for different parameters.

Behavioral tests. All animals used in the TC* binding assays were first washed twice in fresh culture medium and placed together with $2 \mathrm{ml}$ of medium into a $5 \mathrm{ml}$ beaker, the bottom of which was lined with ruled filter paper. Two to $18 \mathrm{hr}$ later most of these animals had settled to the bottom of the beaker and many had become attached to the filter paper. One of each pair of beakers was then mounted in a holder attached to the armature of a solenoid with a calibrated displacement. The armature was displaced $0.25 \mathrm{~mm}$ once each $\mathrm{min}$ for $1 \mathrm{hr}$ to produce habituation. Contractions of individual Stentor in response to these mechanical stimuli were observed through a stereomicroscope.

Binding assays. Six to 15 Stentor were then dislodged from the filter paper in the $5 \mathrm{ml}$ beakers and pipetted into $10 \mathrm{ml}$ of culture medium containing $0.1,2,4,8$, or $16 \mathrm{mM} \mathrm{KCl}$. They were washed with 3 additional $10 \mathrm{ml}$ washes of the appropriate $\mathrm{KCl}$ medium, concentrated into $0.975 \mathrm{ml}$ of medium, and cooled to $15-15.5^{\circ} \mathrm{C}$. Incubation was initiated with the addition of $25 \mu \mathrm{l}$ of $0.6 \mathrm{mM} \mathrm{TC}^{*}$ and continued for 2 min. Cells were then washed free of excess $\mathrm{TC}^{*}$ by manual pipetting through four $10 \mathrm{ml}$ washes of culture medium. Individual cells were pipetted from the last wash onto DEAE filter paper disks, which were then dried. Circles, $3.2 \mathrm{~mm}$ in diameter and centered on the remains of an individual cell, were punched from the filter paper disk and counted at $92 \%$ efficiency on a Packard TriCarb scintillation counter. Three circles containing no cellular remains were also punched from each disk to provide a measure of background activity. The same procedure was followed for unhabituated cells. Occasional cells (19 of 382) produced counts per minute that were more than 2 sample standard deviations in excess of the sample mean, and their data were discarded for reasons stated previously (Wood, 1985).

\section{Results}

\section{Electrophysiological properties}

During the sequence of 15 combined mechanical and electrical stimuli, receptor potential amplitudes decreased exponentially to an average amplitude on trial 15 of $37 \%$ of the average amplitude on trial $1(n=13)$ (Fig. 1). This decrement is comparable to the decrements in receptor potentials and receptor currents reported previously when using such combined stimuli (Wood, 1988). Cells voltage-clamped to $-50 \mathrm{mV}$ after the stimulation sequence produced receptor currents that were $35 \%$ of the prestimulation values $(n=12)$.

Throughout the sequence of habituating stimuli, the membrane resting potential of the 13 cells remained constant $(-52.1$ $\pm 1.5 \mathrm{mV}$ before habituation to $-51.8 \pm 1.5 \mathrm{mV}$ after habituation). On the other hand, action potential amplitudes fell slightly but significantly $(t(13)=4.33 ; p<0.002)$ from $69.7 \pm$ $2.2 \mathrm{mV}$ before habituation to $60.1 \pm 2.7 \mathrm{mV}$ after habituation. This decline was reversible, since the 8 cells still impaled after the final $15 \mathrm{~min}$ rest period produced action potentials whose amplitudes $(69.2 \pm 2.2 \mathrm{mV})$ were not significantly different $(t(8)$ $=-1.4 ; p>0.05)$ from the action potential amplitudes they produced before habituation $(71.6 \pm 1.7 \mathrm{mV})$. In these 8 cells, input resistances also fell slightly over the course of the experiment to $84 \%$ of their initial value, and this decline was significant $(t(8)=2.55 ; p<0.05)$.

\section{I-V plots}

The I-V plots for mechanoreceptor currents in Stentor are characterized by a linear region between -20 and $20 \mathrm{mV}$ and a 
Figure 1. A, Sample records of mechanoreceptor and action potentials (top traces) elicited from a cell being habituated with a combination of mechanical stimulation and depolarizing current pulses (bottom traces). The receptor potential elicited by the first mechanical stimulus (indicated by the first arrow and $M$ below each current trace) became progressively smaller over the 15 trial habituating sequence. Subsequent to this stimulation, an action potential was elicited by a 1-sec-long current pulse. Near the end of the current pulse, a second mechanical stimulus was applied (again indicated by an arrow and $M)$. The receptor potential elicited by this second mechanical stimulus was produced when the cell was still being depolarized by the current pulse. This second receptor potential decreased very little over the 15 habituation trials. $B$, The average amplitude of the first and second receptor potentials elicited on each of the 15 habituation trials, as normalized by the amplitude of the receptor potential recorded on the first trial $(n=13)$. Mean $\perp$ SE.
A
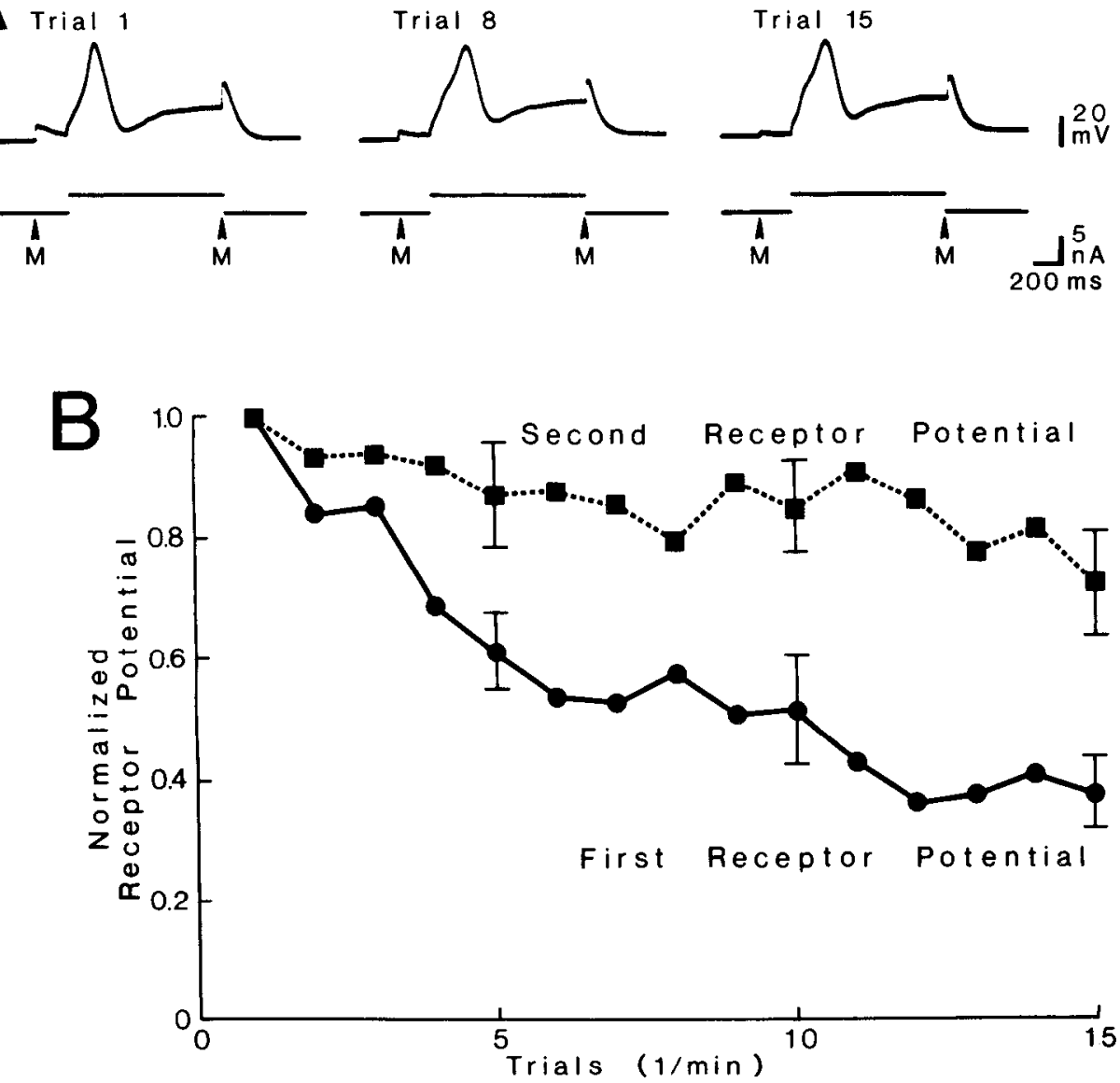

voltage-dependent region between -60 and $-20 \mathrm{mV}$ (Wood, 1982a). Reversal potentials for individual cells were estimated by obtaining receptor current readings at 3 or 4 transmembrane potentials in the linear region of the I-V plot. These data were fitted with a least-squares regression line whose 0 current intercept was taken as the cell's mechanoreceptor current reversal potential. For 8 cells, the reversal potential measured prior to habituation $(21.6 \pm 3.9 \mathrm{mV})$ was significantly different from that measured afterward $(17.2 \pm 5.0 \mathrm{mV})(t(8)=2.52 ; p<$ 0.05 ), but this difference was small (Fig. $2 A$ ). On the other hand, the slopes of the regression lines measured before and after habituation were not significantly different $(t(8)=0.57 ; p>$ 0.05 ). Thus, the linear region of the I-V plot was only marginally altered by the habituation process.

Receptor currents elicited from cells voltage clamped to -50 $\mathrm{mV}$, a value near resting potential, were markedly depressed after habituation relative to their prestimulation values $(t(12)$ $=7.04 ; p<0.001)$ (Fig. $2 A)$. Similarly, receptor currents elicited from cells voltage clamped to -40 or $-60 \mathrm{mV}$ were significantly depressed after habituation relative to prestimulation values $(t(10)=2.17$ and 11.34 , respectively; $p<0.05$ and 0.001 , respectively). On the other hand, a significant depression of receptor currents was not seen when these same cells were voltage clamped to $-30 \mathrm{mV}$ or more positive potentials. Thus, the receptor current depression appears to result from a change in the voltage-dependent characteristic of the mechanoreceptor channel conductance. The voltage dependence of the mechanoreceptor channel conductance, as determined from the data in
Fig. $2 A$, has a maximum slope of $12.3 \mathrm{mV} / e$-fold change for the unstimulated cells, and a maximum slope of $9.6 \mathrm{mV} / e$-fold change for the habituated cells (Fig. $2 B$ ). The voltage dependence of the mechanoreceptor channel conductance is significantly steeper after habituation than before it $(t(8)=2.31 ; p<$ 0.05).

On the basis of this shift in voltage dependence, it is to be expected that receptor potentials elicited at transmembrane potentials depolarized relative to resting potential would habituate less than those elicited at resting potential. This expectation was tested by introducing the return or second mechanical stimulus at a time when the cell's membrane was still being depolarized by the constant current pulse used to elicit the action potential (Fig. 1A). The transmembrane potential at the time of the second mechanical stimulus ranged from $-28.9 \pm 6.7 \mathrm{mV}$ on trial 1 to $-30.7 \pm 6.5 \mathrm{mV}$ on trial 15 . As expected, receptor potentials elicited at depolarized potentials decreased over trials, but not as markedly as those produced at resting potential (Fig. 1). On trial 15 , for example, this difference in decrements was highly significant $(\iota(13)=3.36 ; p<0.005)$. On the other hand, receptor potentials elicited by the second mechanical stimuli underwent marked decrements in amplitude (to 18\%) if they were recorded at resting potential rather than at depolarized potentials. This effect was seen when the current pulse used to elicit the action potential was applied after the second mechanical stimulus. Thus, either action potential production or membrane depolarization during the mechanical stimulus controls the degree of decrement observed. 

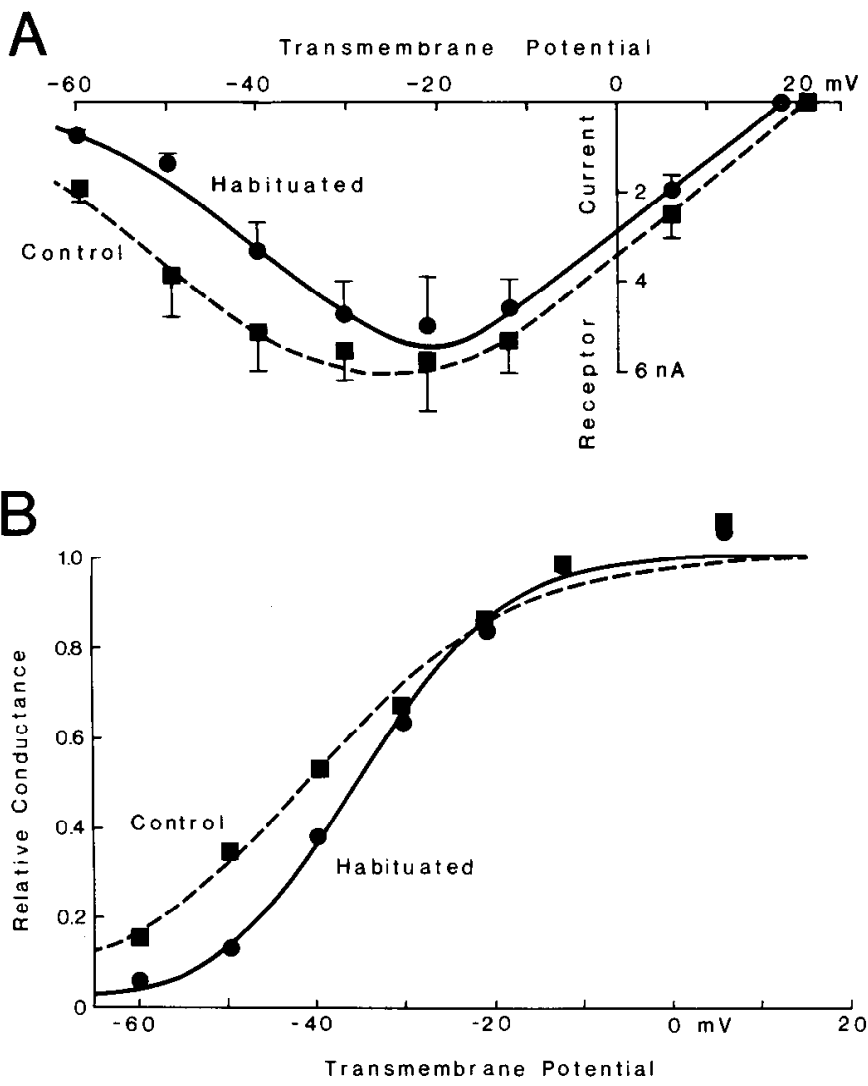

Figure 2. A, I-V plots of mechanoreceptor currents elicited from voltage-clamped cells by mechanical stimuli applied $800 \mathrm{msec}$ after stepping the transmembrane potential to the values indicated along the abscissa. The mechanical stimulation sequence that generated the control data was begun no sooner than $30 \mathrm{~min}$ after microelectrode penetration to insure that the animal had recovered from any habituating effects of the penetration. Test stimuli were applied only once every $3 \mathrm{~min}$. Animals were then habituated by the procedure shown in Figure $1 \mathrm{~A}$. A second set of test stimuli was then applied following the sequence used in the initial test sequence $(n=8-12$ per point $)$. Mean \pm SE. $B$, Voltage dependence of the mechanoreceptor conductance as determined from the data for control and habituated animals shown in $A$. The curves are the best least-squares fit of the Boltzmann equation to the data points.

\section{$T C^{*}$ binding}

As previously stated, $\mathrm{TC}^{*}$ appears to bind selectively to the $\mathrm{U}$ form of the mechanoreceptor channels (Wood, 1985). Since the voltage dependence of the mechanoreceptor channels changes during habituation and therefore the proportion of channels in the $\mathrm{U}$ form should change, it is to be anticipated that $\mathrm{TC}^{*}$ binding to mechanoreceptor channels should change during habituation. This possibility was tested by measuirng TC* binding to both control and habituated cells depolarized to different levels. The level of depolarization was manipulated by varying the concentration of $\mathrm{KCl}$ in the external medium $(0.1,2,4,8$, and $16 \mathrm{~mm}$ ) and estimated from the concentration-dependence function previously derived (Wood, 1982a). Habituation was produced by repetitive $1 / \mathrm{min}$ mechanical stimulation with response probabilities decreasing from 1.0 to 0.96 on the first trial to 0.15 to 0.20 on trials 51 to 60 for the 5 habituated groups.

$\mathrm{TC}^{*}$ binding was highest in both habituated and control groups when the external medium contained $2 \mathrm{~mm} \mathrm{KCl}$ or less and decreased progressively as $\mathrm{KCl}$ concentration increased (Fig. 3). Two-way analysis of variance revealed that $\mathrm{TC}^{*}$ binding was

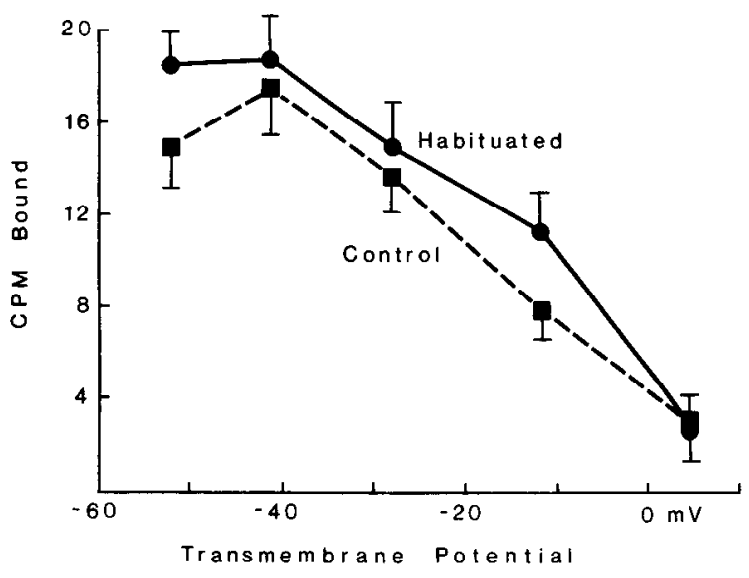

Figure 3. Counts per minute of TC* bound to living Stentor that were bathed in culture medium containing varying concentrations of $\mathrm{KCl}$ during the $\mathrm{TC}^{*}$ incubation. The transmembrane potentials were estimated to be $-52 \mathrm{mV}(0.1 \mathrm{~mm} \mathrm{KCl}),-42.5(2 \mathrm{~mm}),-27.6(4 \mathrm{~mm})$, $-11.7(8 \mathrm{mM})$, and $4.5(16 \mathrm{mM})$, based on data reported previously (Wood, 1982a). Habituated animals were given sixty $1 / \mathrm{min}$ mechanical stimuli prior to TC* incubation ( $n=21-58$ per data point). Mean \pm SE.

significantly affected by external $\mathrm{KCl}$ concentration $(F(4,210)=$ $52.0 ; p<0.001)$ presumably because of the depolarizing action of these solutions. More importantly, habituated animals bound significantly more TC* than did controls $(F(1,210)=5.11 ; p<$ 0.025 ). The amount of $\mathrm{TC}^{*}$ bound to habituated animals was significantly greater than that bound to control animals only at the $0.1 \mathrm{~mm}$ concentration $(t(1)$ Scheffe test $=3.53 ; p<0.05)$.

On the basis of the conductance data displayed in Figure $2 B$, habituated cells should have 1.23 times as many channels in the $U$ form as unstimulated cells at their $-52 \mathrm{mV}$ resting potential. By comparison, habituated cells bound 1.23 times as much $\mathrm{TC}^{*}$ as control cells at resting potential. For cells depolarized to $-42.5 \mathrm{mV}$, the ratio predicted from the conductance data is 1.31 , while the observed ratio is 1.06 . At $-27.6 \mathrm{mV}$ the predicted ratio is 1.13 , and the observed ratio is also 1.13 . At $-11.7 \mathrm{mV}$ the predicted ratio is 1.00 , and the observed ratio of 1.50 is somewhat larger. At $4.5 \mathrm{mV}$ the difference between habituated and control cells disappears both in the conductance data and in the binding data. Thus, while considerable variability is evident, the ratios obtained from the binding data are of the same order of magnitude as those predicted from the conductance measurements.

\section{Discussion}

During the course of repetitive stimulation that combined mechanical stimulation with an action potential elicited by a depolarizing current pulse, the elicited mechanoreceptor potentials progressively decreased in amplitude (Wood, 1988; Fig. 1). Receptor currents recorded from cells at their resting potential also showed a pronounced decrement after this form of stimulation (Wood, 1988; Fig. 2A). These alterations in mechanoreceptor function appear to be the principal cause of habituation of the behavioral response.

In the studies reported above, the amplitude of the elicited action potentials also decreased significantly during the course of the repetitive stimulation. It is unlikely that this action potential decrement contributes strongly to behavioral habituation. This conclusion follows from the observation that action potential amplitudes did not decrease, but behavioral habit- 
uation and pronounced receptor potential decrements did occur when Stentor were stimulated with mechanical stimuli alone at room temperture (Wood, 1971, 1988). Secondly, the action potential decrements observed in this study (14\%) are small relative to the receptor potential decrements $(65 \%)$. Lastly, animals with diminished action potential amplitudes produced observable contractions; hence, a reduction in action potential amplitude cannot explain a decrement in contraction probability. Similarly, the reduction of input resistance observed at the end of these experiments is unlikely to be a major contributor to behavioral habituation. A change in input resistance was not observed after habituation in the previous electrophysiological studies (Wood, 1988), where the number of applied stimuli was fewer and the length of the stimulation period was shorter. Even in the present studies the decrement in input resistance $(16 \%)$ was small compared to the receptor potential decrement. Thus, changes in mechanoreceptor function must be the primary, if not the only, basis of behavioral habituation.

The decrement in receptor potential amplitude appears to be directly due to the decrement in receptor current amplitude, since the 2 changes occur simultaneously and are of the same magnitude. Mechanoreceptor currents in Stentor result from transient increases in membrane conductance and hence are governed by the equation,

$$
I_{\mathrm{r}}=g_{\mathrm{r}}\left(V_{\mathrm{m}}-V_{\mathrm{rev}}\right),
$$

where $g_{r}$ is a function of transmembrane potential and time. While the resting potential, $V_{\mathrm{m}}$, did not change significantly during habituation, the mechanoreceptor reversal potential, $V_{\text {rev }}$, did change significantly. However, this change in $V_{\text {rev }}$ resulted in a reduction of the driving force term by only $6 \%$, whereas the mechanoreceptor current, $I_{\mathrm{r}}$, decreased by $65 \%$. Conversely, the $64 \%$ decrease in $g_{\mathrm{r}}$ (Fig. $2 B$ ) appears sufficient to explain the decrease in $I_{\mathrm{r}}$.

The observed decrease in $g_{\text {r }}$ at resting potential could be attributable to a decrease in the total number of mechanoreceptor channels or an alteration in their voltage dependence. Between the transmembrane potentials of -20 and $20 \mathrm{mV}$ all mechanoreceptor channels should be in the $\mathrm{R}$ form; hence, the slope of the $I_{\mathrm{r}}-V$ plot in this voltage range should be proportional to the total number of functional mechanoreceptor channels. The constancy of this slope before and after habituation implies that habituated cells have the same total number of functional mechanoreceptor channels as unstimulated cells. A general loss of functional mechanoreceptor channels is also counterindicated because there was an increase in $\mathrm{TC}^{*}$ binding to habituated cells as compared to control cells.

Conversely, the greater voltage dependence of the mechanoreceptor conductance observed in habituated cells compared to unstimulated cells (Fig. $2 B$ ) would result in there being a larger proportion of the mechanoreceptor channels in the $U$ form in habituated cells than in unstimulated cells. The smaller decrement observed in receptor potentials elicited from cells depolarized to approximately $-30 \mathrm{mV}$, as compared to the decrements observed in these same cells at resting potential, can also be attributed to this shift in voltage dependence. Lastly, the larger amount of TC* bound to habituated cells at the $-52 \mathrm{mV}$ resting potential than to control cells also suggests that more of the mechanoreceptor channels are in the $U$ form in habituated cells. With a larger proportion of their mechanoreceptor channels in the $\mathrm{U}$ form, habituated cells would have fewer channels open in response to a mechanical stimulus and hence produce a smaller $I_{\mathrm{r}}$.

The increased voltage dependence of the mechanoreceptor conductance can be most easily explained by assuming that the charge on the mobile gating element has increased during the habituation process. The observed change in conductance corresponds to a 1.3-fold increase in charge on the gating element. Such a change could be produced by phosphorylation or any other covalent modification that results in an alteration of gating element charge. $\Lambda$ nother less likely possibility is that the difference in position of the gating element between the $U$ and $R$ forms is reduced in habituated animals.

The mechanoreceptor channel of Stentor appears to be another example on a growing list of voltage-dependent channels that are modified during simple learning processes. Other members of this list include (1) the $\mathrm{Ca}^{2+}$ channel of Aplysia sensory neurons during habituation (Klein et al., 1980), (2) the serotoninsensitive $\mathrm{K}^{+}$channel of Aplysia sensory neurons during sensitization (Klein and Kandel, 1980; Siegelbaum et al., 1982), (3) the A channels of Hermissenda type B photoreceptors during conditioning (Alkon et al., 1982), and (4) possibly the delayed rectifier of Aplysia tail sensory neurons (Baxter and Byrne, 1986). The analysis of habituation in Stentor indicates that the mechanoreceptor channel of this organism is subject to modification exactly because it is voltage-dependent.

\section{References}

Alkon, D. L., I. Lederhendler, and J. J. Shoukimas (1982) Primary changes of membrane currents during retention of associative learning. Science 215: 693-695.

Baxter, D. A., and J. H. Byrne (1986) Serotonin-modulated membrane currents in Aplysia tail sensory neurons. Soc. Neurosci. Abstr. 12: 765 .

de Peyer, J., and H. Machemer (1978) Hyperpolarizing and depolarizing mechanoreceptor potentials in Stylonychia. J. Comp. Physiol. 127: 255-266.

Hodgkin, A. L., and A. F. Huxley (1952) A quantitative description of membrane current and its application to conduction and excitation in nerve. J. Physiol. (Lond.) 117: 500-514.

Klein, M., and E. R. Kandel (1980) Mechanism of calcium current modulation underlying presynaptic facilitation and behavioral sensitization in Aplysia. Proc. Natl. Acad. Sci. USA 77: 6912-6916.

Klein, M., E. Shapiro, and E. R. Kandel (1980) Synaptic plasticity and the modulation of the $\mathrm{Ca}^{2+}$ current. J. Exp. Biol. 89: 117-157.

Siegelbaum, S. A., J. S. Camardo, and E. R. Kandel (1982) Serotonin and cyclic AMP close single $\mathrm{K}^{+}$channels in Aplysia sensory neurones. Nature 299: 413-417.

Wood, D. C. (1970) Parametric studies of the response decrement produced by mechanical stimuli in the protozoan, Stentor coeruleus. J. Neurobiol. 1: 345-360.

Wood, D. C. (1971) Electrophysiological correlates of the response decrement produced by mechanical stimuli in the protozoan, Stentor coeruleus. J. Neurobiol. 2: 1-11.

Wood, D. C. (1975) Protozoa as models of stimulus transduction. In Aneural Systems in Neurobiology, E. Eisenstein, ed., pp. 5-23, Plenum, New York.

Wood, D. C. (1982a) Membrane permeabilities determining resting, action, and mechanoreceptor potentials in Stentor coeruleus. J. Comp. Physiol. 146: 537-550.

Wood, D. C. (1982b) A chemical model for the binding of $d$-tubocurarine to voltage-dependent channels in the protozoan, Stentor coeruleus. Soc. Ncurosci. Abstr. 8: 908.

Wood, D. C. (1985) The mechanism of tubocurarine action on mechanoreceptor channels in the protozoan Stentor coeruleus. J. Exp. Biol. 117: 215-235.

Wood, D. C. (1988) Habituation in Stentor: A response-dependent process. J. Neurosci. 8: 2248-2258. 